\section{G-CSF-Prophylaxe in der Praxis}

Nach einer Chemotherapie wird für einige Patienten die Behandlung mit Granulozyten-stimulierendem Faktor (G-CSF) empfohlen, um einer febrilen Neutropenie (FN) und den damit verbundenen Komplikationen vorzubeugen. Doch wie gut ist diese Prophylaxe in der Praxis implementiert?

Kome ommt es bei einer Neutropenie nach Chemotherapie zu Fieber, kann dies das erste Anzeichen einer schweren Infektion ein. Eine FN ist definiert als Rückgang der absoluten Neutrophilenzahl auf $<1.000 / \mathrm{mm}^{3}$ und eine Einzelmessung der Körpertemperatur von $>38,3^{\circ} \mathrm{C}$ oder eine Körpertemperatur von konstant (länger als 1 Stunde) $\geq 38^{\circ} \mathrm{C}$. Das Sterberisiko von Patienten mit FN, die in die Klinik eingewiesen werden, liegt bei 9,5\% - bei Komorbiditäten ist es noch höher.

Durch die Gabe von G-CSF nach einer Chemotherapie erholen sich die Neutrophilen schneller, wodurch eine FN verhindert werden kann. In den amerikanischen, europäischen und deutschen Leitlinien der Fachgesellschaften wird die Prophylaxe mit G-CSF bei hohem Risiko oder bei intermediärem Risiko mit weiteren Risikofaktoren empfohlen.

In einer retrospektiven Untersuchung wurden 195 deutsche Hämatologen, Gynäkologen und Pulmologen mit Schwerpunkt Onkologie aus Praxis und Klinik dazu befragt, wie oft sie G-CSF bei Patienten mit malignem Lymphom, Brustkrebs und Lungenkarzinom angewendet hatten. Dokumentiert wurden die Daten von 666 Lungenkarzinompatienten, 286 Patienten mit malignem Lymphom und 976 Patientinnen mit Brustkrebs. Sie durchliefen insgesamt 7.805 Chemotherapiezyklen (jeweils 3-9 Zyklen). 85,1\% der befragten Ärzte gaben an, die Prophylaxe mit G-CSF angewendet zu haben.

Die Leitlinien-Adhärenz bei allen Chemotherapiezyklen mit hohem FNRisiko lag für Patienten mit Lungenkarzinom bei $15,4 \%$, mit malignem Lymphom bei $84,5 \%$ und mit Mammakarzinom bei $85,6 \%$ - bei allen Chemotherapiezyklen mit mittlerem FN-Risiko betrug sie je $38,8 \%, 59,4 \%$ und $49,3 \%$. Ein übermäßiger Gebrauch von G-CSF (ohne Vorliegen zusätzlicher Risikofaktoren) erfolgte bei 7,2\% der Lungenkarzinom-Zyklen, 16,8 \% der Lymphom-Zyklen und 17,6\% der Brustkrebs-Zyklen.
Unter den Pulmologen hatten jene, die kürzer als 22,5 Jahre praktizierten, eine höhere Leitlinien-Adhärenz. Höher war die Adhärenz bei Pulmologen mit mehr als 22,5 Jahren Berufserfahrung, wenn sie an weniger als 1,5 internationalen Konferenzen pro Jahr teilnahmen. Bei den anderen Spezialisten bestand eine größere Adhärenz, wenn sie 8 Jahre oder weniger Berufserfahrung hatten.

Fazit: Die Akzeptanz und Adhärenz zur leitliniengestützten Anwendung von GCSF als FN-Prophylaxe erwies sich in der Befragung als unterschiedlich. Die Ärzte überschätzen nach Ansicht der Forscher ihre Leitlinien-Adhärenz.

Kathrin von Kieseritzky

Link H et al. Adherence to granulocyte-colony stimulating factor (G-CSF) guidelines to reduce the incidence of febrile neutropenia after chemotherapy-a representative sample survey in Germany. Support Care Cancer. 2016;24(1):367-76.

\section{Kommentar von Dr. Marschner:}

Auf den ersten Blick ist es enttäuschend, wie gering die Adhärenz der Onkologen an die Leitlinien zur Prophylaxe der FN mit risikoadaptiertem Einsatz von G-CSF-Formulierungen zu sein scheint. Zumal die Adhärenz für alle drei Indikationen mit zunehmender Erfahrung der Onkologen weiter abnimmt. Spezialisten für die Therapie des Lungenkarzinoms mit über 22,5 Jahren Erfahrung zeigen dabei die geringste Adhärenz. Der absolute Einsatz von G-CSF Präparaten liegt für alle drei Indikationen teilweise deutlich unter den Leitlinienempfehlungen. Was bedeutet dies für die Versorgungsqualität oder für die FN-Leitlinien?

Harte FN-Risiken (> $20 \%$ oder $10-20 \%$ plus zusätzliche Risikofaktoren) basieren auf den verabreichten Chemotherapie-Regimes. Es deutet sich an, dass die bekannten "weichen" Risikofaktoren (Alter > 65 Jahre, weiblich, ungünstiger ECOG [Eastern Cooperative Oncology Group]-Performancestatus, schlechter Ernährungszustand, $\mathrm{Hb}<12 \mathrm{~g} / \mathrm{dl}$, Komorbiditäten etc ...) bei den befragten, erfahrenen Ärzten eine wesentliche, therapeutisch entscheidende Rolle spielen. Um dies besser beurteilen zu können, wäre die Listung der Ärztecharakteristika (Basis zur Analyse des Ärzteverhaltens) und der eingesetzten Therapieregimes und Patientencharakteristika (Basis für die Analyse der Therapierisiken) wünschenswert gewesen. Daten zum Erfolg der FN-Behandlung wurden nicht erhoben und waren angesichts der retrospektiven Studienmethodik auch nicht Ziel dieser Untersuchung.

Die entscheidende Frage, ob es Unterschiede hinsichtlich des Erfolgs der FN-Prophylaxe zwischen leitlinienkonformen G-CSFAnwendern und G-CSF-Vermeidern gibt, ist durch die Studiendaten nicht beantwortbar. Die Divergenz zwischen den Leitlinien und der Versorgungsrealität sollte Anstoß sein, die FN-Leitlinien nicht allein auf Basis randomisierter, kontrollierter Studien, sondern auch auf Grundlage der Versorgungsforschung zu erstellen. Wenn sich bis zu $85 \%$ der verantwortlichen, erfahrenen Onkologen nicht an die FN-Leitlinien halten, wäre im Rahmen von sorgfältig geplanten, reprä-

\section{"Muss die Adhärenz verbessert oder die Leitlinie an die klinische Realität angepasst werden?"}

sentativ und prospektiv angelegten Versorgungsforschungsprojekten zu überprüfen, ob dies die Versorgungsqualität (Häufigkeit von FN) beeinflusst oder nicht. Die Frage, ob die ärztliche Adhärenz an die Leitlinien verbessert oder die Leitlinien an die Versorgungsrealität angepasst werden sollten, ist nicht beantwortet.

Es ist der Verdienst von Hartmut Link und Kollegen, dass sie die fehlende Adhärenz an die FN-Leitlinien retrospektiv aufgezeigt haben. Ob dies klinisch relevant ist, kann nur die prospektive, zusätzliche Analyse des Erfolgs der GCSF-Prophylaxe zeigen. Dies ist meines Erachtens nach die wichtigste Erkenntnis aus der Studie und macht ihren Charme aus.

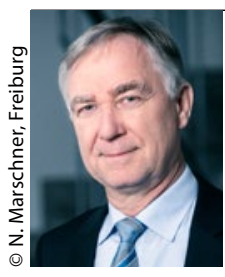

Dr. med.

Norbert Marschner Praxis für interdisziplinäre Onkologie \& Hämatologie, Freiburg norbert.marschner@ iomedico.com 